\title{
Burkholderia cenocepacia
}

National Cancer Institute

\section{Source}

National Cancer Institute. Burkholderia cenocepacia. NCI Thesaurus. Code C86223.

A species of aerobic, Gram-negative, rod shaped bacteria assigned to the phylum

Proteobacteria. This species is non-spore forming, motile, catalase and oxidase positive, indole negative and does not hydrolyze esculin or starch. B. cenocepacia is naturally found in soil, water and vegetation and is capable of degrading industrial waste, herbicides, and vegetation as well as producing many antibacterial and antifung al agents. In humans, this species is pathogenic and associated with cystic fibrosis. 